\title{
FUNCION Y ESTRATEGIAS DE LA ESCRITURA EN SARMIENTO
}

\author{
Mónica Elza Scarano \\ Universidad Nacional de Mar del Plata
}

RESUMO

\begin{abstract}
Com este artigo se pretende uma leitura da obra de Domingo Faustino Sarmiento, figura política e literária da Argentina do século XIX, com o intuito de lê-lo em suas contradiçōes, em seu afā programático e em sua visão sin. tética e intuitiva da realidade nacional. O centenário de sua morte em 1988 deu a oportunidade para que, nesta década que ora finda, o célebre autor de Facundo fosse recordado tanto como personalidade histórica significati. va, testemunha de uma época e de um modo de pensar e fazer literatura.
\end{abstract}

La ocasión del Centenario de la muerte de Sarmiento nos da la oportunidad de recordarlo en tanto personalidad significativa de nuestra historia, testimonio de una época y de un modo de pensar y hacer literatura. Es una ocasión propicia para poner en sus justos términos la cuestión acerca de Sarmiento: leer su sistema de ideas y su producción literaria e interpretarlas desde una nueva perspectiva, propia y original. Desde este contexto de recepción - actual y nuestro -, se nos plantea el desafío de superar divisiones maniqueas y abordar la literatura y las ideas de Sarmiento, sin comprometernos ni en una apología acrítica ni en una condena ciega y prejuiciosa, para rescatar lo que de él pervive como error o como acierto entre nosotros y desentrañar las claves politicas, sociales, estéticas e ideológicas que lo condicionan, y de este modo poder comprendernos mejor a nosotros mismos para, a partir de allí, elaborar nuestro proyecto nacional, con un mayor grado de ajuste y de fidelidad hacia nuestra realidad.

Me propongo releer la producción literaria de Sarmiento desde el concepto de la función que le asigna a la escritura y 
las estrategias textuales que se detectan en los textos más logrados de cada etapa. Es posible distinguir tres momentos en el corpus total de la producción literaria sarmientina. Dejaré de lado la breve experiencia periodistica, entre el primer y segundo exilio en tierra chilena (julio y agosto de 1839), lapso durante el cual se publicó su periódico El Zonda en San Juan, y en el que están presentes, aunque potencialmente, las ideas que desarrollará pocos años después en sus escritos, artículos y libros.

La primera etapa comprende lo mejor de los escritos de Sarmiento, producidos en Chile, desde los primeros artículos periodísticos publicados en El Mercurio de Valparaíso, en 1841, hasta su regreso a la Argentina para unirse al ejército de Urquiza, con la intención de poner fin al gobierno de Rosas, en 1851. La critica en general coincide en identificar a este período con el "ciclo de Sarmiento escritor".

Los textos producidos entre estas fechas pueden ser considerados dentro de los que podríamos denominar "el discurso del exilio". Indirectamente privado de la libertad de seguir escribiendo el semanario El Zonda en su provincia natal, mediante las astucias del gobernador de San Juan, Nazario Benavides, y sin la posibilidad de pensar libremente, ante las condiciones para él adversas que le ofrecía el país en esos tiempos, Sarmiento se ve obligado a cruzar por segunda vez la frontera para refugiarse en Chile, tierra a la que admira profundamente por su grado de civilización y libertad. Sus textos chilenos se difundirán en distintos ámbitos: El Diario: a los tres meses de radicarse alli, logra que se le publique un artículo acerca de la batalla de Chacabuco en El Mercurio de Valparaíso ("12 de febrero de 1817", El Mercurio, 11-II-1841), firmado con el seudónimo "Un teniente de artillería". Su labor periodística no se limitará a la producción de incontables artículos de crítica, especialmente teatral, además de política, polémica social, arte, filosofía, estética, sino también a la fundación de periódicos (El Progreso de Santiago de Chile, El Heraldo Argentino, entre otros). Por este medio logra ingresar a la élite letrada y encuentra un canal de comunicación para alcanzar una considerable cantidad de lectores.

- Lo público - académico: se incluyen en este espacio los escritos pedagógicos y educativos (informes, prospectos, memorias) elaborados en un marco institucional. En ellos desplegará su programa alfabetizador (planes educativos, nuevos programas, proyecto de reforma ortográfica) que persigue la meta de "convertir al bárbaro en lector", paso imprescindible para acceder a la posibilidad de moderniza- 
ción del pais, que Sarmiento vislumbraba como elemento integrante del imperativo histórico del progreso.

- Campo editorial: en 1843 publica en hojas sueltas, en busca de una circulación rápida y más limitada el escrito autobiográfico Mi defensa. Su famoso Facundo aparece primero como folletín por entregas en el diario El Progreso en 1845 y en el mismo año aparece como libro - lo mismo ocurrió con Apuntes biográficos (Vida de Aldao). En 1849 aparecen los dos tomos de Viajes por Europa, Africa y América, en 1850 Argirópolis y luego Recuerdos de provincia.

Los textos del exilio elaboran, en su conjunto, un programa cultural, que mediante diferentes claves y utilizando diversas estrategias discursivas - entendidas como medios literarios que consisten en modos especificos de constituir el discurso con una determinada intención encubierta - persiguen el objetivo de ser eficaces.

Me detendré en particular en dos textos de esta primera etapa: Facundo y Recuerdos de provincia. Como otros textos de este autor, ambos se encuentran estructurados en torno a dos ejes principales: lo autobiográfico/politico (antirrosismo).

\section{La estrategia autobiográfica.}

El primer escrito autobiográfico de Sarmiento, Mi defensa, refleja ya la voluntad de representación de una estrategia defensiva y de autojustificación, que en este caso refleja la polémica entre Sarmiento y el ex-cónsul de Chile en San Juan, Domingo S. Godoy. Este texto compondrá la primera parte de las memorias politicas y literarias del autor sanjuanino.

La segunda defensa de Sarmiento serán sus Recuerdos de provincia, en respuesta a las acusaciones y el descrédito promovido por parte del gobierno de Rosas hacia su persona. En las palabras que dirige a sus compatriotas, antes de comenzar la narración de sus antepasados, escribe:

Ardua tarea es, sin duda, hablar de sí mismo y hacer valer sus buenos lados sin suscitar sentimientos de desdén, sin atraerse sobre si la critica, y a veces con harto fundamento pero más arduo aún es consentir la deshonra, tragarse injurias y dejar que la modestia misma conspire en nuestro daño...)R. de p., p. 27)'1.

2 Dominco Faustino Sarmicnto. Recuerdos de provincia, en: Obras, tomo III. Bs. As.: Luz del Dia, 1498, p. 27. 
Este escudo defensivo no fue bien recibido por sus contemporáneos, quienes consideraron impudorosa la frecuencia con que Sarmiento hablaba de si mismo, particularmente a partir de la lectura nada concesiva de Juan Bautista Alberdi, quien adivinó que, tras el pretexto de presentar una autodefensa contra la política de Rosas, se ocultaba una operación mucho más ambiciosa y compleja, en ese tiempo de programas y propuestas de organización nacional. Se trata, de acuerdo con la lectura de Alberdi, de atraer la atención de los lectores sobre sí mismo, para exponerse y presentarse como "héroe cultural", única y verdadera alternativa frente a Rosas y el régimen de los caudillos. Se adelanta, por este medio, la figura de un candidato, aspiración formulada explícitamente por Sarmiento, quien hacia 1849 se daba diez años para alcanzar a ocupar el puesto de Rosas, en el gobierno de la República².

Lo autobiográfico se carga aquí de distintas motivaciones: a la política se agrega la psicológica (egotismo, titanismo romántico "Don Yo"); y en el orden de lo personal y su contexto socio-cultural, la de hacer la vindicación de sus méritos no reconocidos, que da cuenta del horizonte ideológico de su mundo intelectual (autodidactismo, lecturas, maestros).

\section{La estrategia biográfica.}

En uno de sus artículos chilenos, escrito en 1842, Sarmiento recomienda la difusión en nuestros pueblos de las vidas de hombres célebres ${ }^{3}$. La afición por la biografía se explica en Sarmiento por su inclinación por un criterio de "excepcionalidad" inscripto en la teoria del "grande hombre", cuya vida merece ser presentada y difundida entre el pueblo como un ejemplo de vida pública, un arquetipo de civilización, libertad y de independencia, que contribuirá a purificar las costumbres y a promover los valores que encarna.

Hay en Sarmiento una concepción didáctico-moral. Leemos en Recuerdos de provincia:

Gusto, a más de esto, de la biografía. Es la tela más adecuada para estampar las "buenas ideas"; ejerce el que las escribe una especie de judicatura, castigando el vicio triunfante, alentando la virtud oscurecida... ${ }^{4}$.

2 Es conocida la anécdota de que en la época circulaba una foto con el retrato de Barmiento, que tenia una inscripción que decia aproximadamente: "Domingo Faustino Sarmiento, futuro Presidente de la República Argentina".

3 D.F. Sarmiento, "De las blografias", El Mercurio, 20.III.1842, en: Obras, I, p. 182.

4 Recuerdos..., p. 27 
Se propone la exposición de ejemplos concretos de una vida, no sólo por su ejemplaridad moral, sino también por su eficacia como instrumento didáctico.

Sarmiento escribe y lee biografias con gusto. Esta forma discursiva lo acompañará a lo largo de las distintas etapas de su producción: desde los Apuntes biográficos (Vida de Aldao), hasta la . Vida de Francisco J. Muñiz (1885) y la Vida de Dominguito (1886), pasando por Facundo, El Chacho, Franklin, Iincoln, entre otros.

En Facundo se presenta a Quiroga como individuo representativo con el fin de perfilar una ejemplaridad histórica de orden inverso. Invocado como un medio de conocimiento histórico y un instrumento apto para descifrar enigmas, el tipo o individuo elegido condensa el significado de una época y hasta de una región geográfica precisa.

En Recuerdos... hará de su propio destino, un destino histórico, representativo, conciente de si y portador de un sentido histórico dentro del marco filosófico del historicismo providencialista. Sarmiento se presenta como heredero de un linaje y la vez como un "self - made man". En su vida le parece ver retratada la pobre América del Sur. Asi su vida será propuesta como un "espejo" de la vida de América del Sur, en la tentativa por constituir un nuevo orden. Sin abandonar la teoría romántica y subjetiva de la historia nacional, contemplada desde los "grandes hombres" y los "tipos fundamentales", en primer lugar se insinúan presagios y anticipaciones que adelantan una "historia cifrada". A continuación la narración autobiográfica en primera persona del singular anunciará el desenlace antes que la historia cifrada. El contraste y compenetración entre historia nacional y biografía personal se hacen presentes en el discurso a través del contrapunto rítmico entre el pasado y el presente. Tanto la historia del pais, como la biografía o autobiografía del candidato están, del mismo modo, subordinados a la estrategia de persuasión, que pretende presentar al candidato como un individuo capaz de brindar soluciones para los problemas argentinos y al mismo tiempo presentarlo como única alternativa rival de Rosas.

A la estrategia ensayistica que domina en la mayor parte de los textos de Sarmiento, se agrega la heterogeneidad esencial de los mejores escritos del autor - presente ya en Facundo, pero agudizada em Recuerdos..., donde se manejan distintos modelos literarios, con una notable disparidad temática y retórica, hasta caer en la acumulación y la abundancia. 


\section{La estrategia programática-politica.}

La producción literaria de Sarmiento compone en su totalidad lo que podriamos llamar "un discurso civilizador", que implica una determinada visión de la realidad, $\therefore$ que : se detendrá en establecer un análise de la dualidad de elemen. tos opuestos que se detecta en ella: civilización - barbarie. El análisis de la realidad que, se realiza em Facundo no es de ningún modo "inocente", sino que responde a un programa concreto.

En el capitulo segundo de ese texto trata de dibujar el "tipo nacional" mediante la técnica de cuadros vivos ("tableaux vivants"), con la intención de "ordenar" el caos y hacer más comprensible la realidad. La función de "explicar". la realidad y desentrañar las causas genéticas de la misma (determinismo mesológico) domina la primera parte del Facundo. Pero a ese deseo se superpone con mayor ansiedad. la voluntad de representación que apunta a un programa político (por estos hechos y factores la realidad es ésta, por eso la solución es tal otra).

A pesar de la indisolubilidad de la dicotomía civilizaciónbarbarie, la dupla no es absoluta ni monolítica. Mediante estrategias propias de un relato ficticio, Sarmiento introduce una dosis de "ficcionalización" con el objeto de forzar a la realidad, con lo que tergiversa la verdad histórica, generán. dose un conflicto entre la verdad histórica y la verdad literaria. Sin embargo, una lectura minuciosa descubre que en este análisis emergen fisuras, que advertirá más que nínguno de sus contemporáneos, Juan B. Alberdi: las contradicciones y la relatividad de los términos de la antinomia, extensibles a su programa. En primer lugar, abundan en sus escritos pasajes que demuestran que tanto la civilización como la barbarie no son realidades absolutas ni puras, sino que se contaminan entre si. Tal vez el texto más significativo y revelador lo encontremos en los Viajes, cuando comprueba que en Paris - centro de su ideal de civilización - también existe miseria, realidades desagradables, es decir "barbarie"s. A partir de ese hecho, Sarmiento descubre su ponto de vista "americano" y al mismo tiempo desplazará su mirada del modelo europeo al modelo norteamericano.

Si se lee el Facundo "entre líneas" se hará evidente que, aún. admitiendo el recurso al didactismo siempre presente en Sarmiento, la dicotomía civilización - barbarie funciona en la escritura de Sarmiento más como consigna, que como

5 Vinjes por Europa, Africa y Ambrica (1845-1847), en: Obras, tomo v. 
esquema descriptivo de la realidad nacional americana. Todo está subordinado a los componentes programáticos y prescriptivos, que apuntan a diseñar un modelo de nación.

Por otra parte, la segunda contradicción se centra en el programa mismo propuesto por Sarmiento: civilizar pero valiéndose de medios bárbaros. Alberdi realiza esta crítica a partir de la comparación del gobierno de Sarmiento en el programa que redactara veinte años antes en el Facundo.

La presentación de la "utopia" sarmientina no está exenta de tensiones: en primer término, la confrontación del mundo deseado y soñado con el mundo real, a la que pretende poner fin con el proyecto de raíz iluminista de reformador ilustrado, reforzado por una fuerte cuota de optimismo providencialista proveniente del romanticismo: civilizar, abrir las mentes, difundir las luces, racionalizar. Este proyecto coincide en gran parte con la propuesta de la "generación del 37" de cortar todo vinculo con la tradición hispánica (antihispanismo, europeismo). En segundo término, existe en Sarmiento una lucha interior entre la sedución estética de lo bárbaro, americano, y esa misma realidad que debe ser suprimida por lo que representa. Tal conflicto es evidente en las páginas dedicadas a retratar al "gaucho malo".

La segunda etapa de la obra literaria sarmientina incluye los escritos posteriores a la derrota de Rosas en Caseros, producidos en su mayoria durante el tercer exilio chileno, luego de su distanciamiento con Urquiza. En este período como en el último, la producción literaria disminuye notablemente, debido a que Sarmiento ejerce la función pública, al represar al pais en (1855 (Jefe del Departamento de Escuelas en Buenos Aires (1856), senador provincial (1858), senador nacional (1860), ministro de Gobierno y Relaciones Exteriores diputado por la Capital a la Convención para la reforma de la Constitución Nacional, gobernador de San Juan (1862), ministro plenipotenciario ante Chile, Perú y los Estados Unidos, Presidente de la Repüblica (1868).

\section{La estrategia polémica.}

Los textos chilenos posteriores a Caseros continuan el "discurso civilizador" iniciado en la etapa anterior, con el agregado de un factor de decisiva importancia: el hecho de disputar el acceso al poder, una vez derrotado Rosas. Se despliega en este periodo una discusión "interna" entre quie. nes se identifican con la "civilización", dentro de um mismo espacio ideológico, en ese momento dominante: el liberal. Ocupa un lugar privilegiado en este enfrentamiento planteado entre dos "discursos civilizadores" la polémica entre Sar- 
miento y Alberdi. Después de romper con Urquiza, Sarmiento llega a Valparaíso y concuerda con Alberdi en mantenerse expectantes por favorecer la unión nacional y la organización constitucional. Al poco tiempo se entera de que Alberdi habia sido nombrado en Paraná enviado diplomático de la Confederación ante el gobierno de Chile, para allegar las opiniones en torno a Urquiza y en contra de Buenos Aires.

Con tal fin se forma el Club de Valparaiso y Alberdi comienza a escribir en El Diario de Valparaíso. En ese año 7852 - Sarmiento publica en Chile Campaña en el Ejército Grande aliado de Sud América, a partir de los boletines escritos durante la guerra contra Rosas. Alli proclama su disidencia con el régimen naciente en el interior argentino bajo la influencia del vencedor de Caseros. Alberdi responde, identificado con Urquiza con las Cartas sobre la prensa y la política militante en la República Argentina, conocidas como Cartas quillotanas. Um mes después Sarmiento contesta con Las ciento y una. Alberdi replicó con Complicidad de la prensa en las guerras civiles de la República Argentina. Ya sancionada la Constitución Argentina, en 1853 Sarmiento dio a conocer los Comentarios de la Constitución de la Confederación Argentina, a los que siguieron los Estudios sobre la Constitución Argentina de Alberdi. Dentro de este marco bibliográfico se desarrolla la polémica, que despliega un debate en torno a la imagen de pais que la facción que monopolizara el poder intentaría construir. La confrontación ideológica se detiene en la diferencia de matices, medios y fines que delinean dos perfiles culturales diversos. La estrategia polémica de Sarmiento consistirá en descalificar a su adversario en lo personal. Para hacer frente a la lectura "destructiva" de Alberdi, quien desarticula a Sarmiento en sus propios escritos, calificándolo como "un caudillo de la pluma", "un gaucho malo de la literatura", el escritor sanjuanino confiesa en primer lugar no aceptar la polémica que el tucumano le promoviera desde El Diario de Valparaiso y le recuerda otros disentimientos que no lograron alterar la amistad entre ambos.

Sin embargo en Las ciento y una, verdadera "autobiogra. fia intelectual", ordena sus pensamientos:

En la olla podrida que ha hecho Ud.. de Argirópolis, Facundo, La Campaña, etc., etc., condimentados sus trozos con la vistosa salsa de su dialéctica saturada de arsénico, necesito poner orden para responder y restablecer cada cosa en su lugar ${ }^{\mathbb{Q}}$.

6 Las ciento y Una (Primera), en: Obras, tomo XV..., p. 123. 
Queda así desmentida la primera afirmación de Sarmiento de abstenerse de polemizar. Para desarticular a sua contradestinatario, Sarmiento recurre a la estrategia de volver las frases de aquel en su contra, aplicándolas a su persona y poniendo en evidencia la incoherencia de su discurso. Utilizando una astuta y sutil ironia, le responde: "A Ud. no se le refuta. Con rectificar lo que Ud. dice, nada queda por hacer", puesto que todo lo que se le ha imputado a Sarmiento, de él mismo (Alberdi) lo sacó el adversario del sanjuanino, o de los jóvenes de Buenos Aires de 1837, de que Alberdi formaba parte ${ }^{i}$. Con distintas estrategias, ambos contendores persiguen el mismo fin: coaccionar para alcanzar el status de "lo verdadero", como efecto de sentido. La mima voluntad de verdad los lleva a entrar en conflicto poniendo en juego su capacidad persuasiva y su voluntad de generar "verdades". En la confrontación, subyace, sin lugar a dudas, la deliberada intención de eliminar desde las argumentaciones del discurso al adversario para allanar los obstáculos que se interponen en el camino del poder. Si bien el tiempo demonstró que buena parte de la razón estaba de parte de Alberdi; la eficacia discursiva de Sarmiento superó rotundamente a su adversario y logró los propósitos ocultos que perseguia lograr ocupar el centro de la discusión, convocar la atención del público lector y capitalizar sus intereses hacia su "Yo". Cuando Alberdi advierte esta argucia de Sarmiento pone fin a la polémica, optando por atacarlo por su flanco más débil: no darle el gusto de que se hable de él.

La última etapa de sus escritos se compone de los textos producidos a partir de sua asunción como Presidente de la República Argentina hasta su muerte (1868-1888). La escritura de Sarmiento adopta en este periodo la forma de un "discurso de rectificación", generado desde la desilusión de contemplar los sueños concretados en la realidad, con desajustes y materializaciones deseadas. La obra más destacada que Sarmiento escribe en esta época es, sin duda, Conflicto y armonias de las razas en América, en que explicita su voluntad de pensar y construir la identidad, no sólo nacional, sino de toda Hispanoamérica.

\section{La estrategia cientifica.}

Los textos de la desilusión pretenden salvar la distancia entre el programa y su realización. Para ello, Sarmiento recurre a la estrategia de "explicar" la realidad americana,

7 Ibidem, p. 124. 
mediante una aproximación a argumentos del discurso científico, que en la segunda mitad del siglo XIX venian ganando mayor eficacia para dar respuesta a los interrogantes en torno a la América del Sur. Con menor poder de convicción y sin alcanzar el grado de sutileza estilística que contienen sus primeros escritos, la escritura de Sarmiento intenta asumir la forma del discurso positivista.

En los "Prolegómenos" del primer volumen de Conflicto... plantea las preguntas básicas, implícitas en sus obras anteriores, que conforman un verdadero aporte para dilucidar el cuestionamiento acerca de la gestación de una conciencia nacional y continental:

Es acaso ésta la vez primera que vamos preguntarnos quiénes éramos cuando nos llamaron americanos, y quiénes somos cuando argentinos nos llamamos.

¿Somos europeus? iTantas caras cobrizas nos desmienten!

iSomos indigenas? Sonrisas de desdén de nuestras blondas damas nos dan acaso la única respuesta. ¿Mixtos? Nadie quiere serlo, y millares que ni americanos ni argentinos quérrian ser llamados.

¿Somos Nación? Nación sin amalgama de materiales acumulados, sin ajuste ni cimiento? ¿Argentinos? Hasta dónde y desde cuándo, bueno es darse cuenta de ello.

Ejerce tan poderosa influencia el medio en que vivimos los seres animados, que a la aptitud misma para soportarlo se atribuyen las variaciones de razas, de especies y aún de género.

Es nuestro ánimo descender a las profundidades de la composición racial de nuestras poblaciones... ${ }^{8}$.

\section{La función social de la literatura.}

Las diferentes estratégias utilizadas a lo largo de toda su producción y la asombrosa heterogeneidad temática que se observa en su escritura confluyen, sin embargo, en una zona que les es común a todos sus textos: la función que se le asigna a la escritura. Sarmiento no concibe a la literatura sino está revestida de utilidad para la sociedad en formación. En su concepción de la escritura, la identifica con la actividad

B Conflicto y armonfas de las razas en Ambrica, en: Obras, tomo XXXVII. D. 23. 
politica. Escribir es un medio para civilizar, una via apta para formalizar um proyecto nacional y proponer un modelo de república, poniendo en marcha, al mismo tiempo la maquinaria de acción que intente transformar la barbarie enquistada en la realidad nacional y americana.

Por esta razón, importa lograr un efecto de eficacia, para generar confiabilidad y llegar a convencer al público lector. Esta idea acerca de la literatura se entiende en relación con la función del intelectual de la época, que cumple el doble rol de hombre de letras y hombre púbiico, lo que se traduce en la escritura en la "contaminación de los distintos discursos" (literario, político, histórico, filosófico, económico, sociológico).

Escribir literatura, pues, es para Sarmiento uma tarea patriótica. Literatura es acción, que incumbe al dominio público; asi se define como escritor social y se incluye dentro de la escuela socialista:

... que no escribe por escribir como la romántica, ni para imitar maquinalmente como la clásica, sino para servir a los intereses de la sociedad?.

Años más tarde definirá su concepción pragmática de la literatura una vez más, en Campaña en el Ejército Grande...:

... Soldado, con la pluma o la espada, combato para poder escribir, que escribir es pensar; escribo como medio y arma de combate, que combatir es realizar el pensamiento ${ }^{10}$.

La brevedad del recorrido por algunos textos y algunos aspectos de la práctica literaria en Sarmiento no impide advertir a simple vista que su figura política y literaria, tanto por su sistema de ideas como por su escritura, se nos presenta hoy, un siglo después de su muerte, como un interrogante fecundo. Tal vez el auge de un revisionismo histórico mal entendido sea la consecuencia de que no revisamos nuestro pasado ni nuestro presente sino a través de nuestros propios prejuicios y pasiones. Se impone entonces para revertir este proceso, la tarea de descubrir a Sarmiento - como lo ha sugerido David Viñas - "ni beato ni demonio", leerlo en sus contradicciones, en su afán programático y en su visión sintética e intuitiva de la realidad nacional. Develarlo y reformularlo desde nuestro presente es hoy nuestro desafio.

9 "Segunda polémica literaria". El Mercurio. 1842, en: Obras, t. I.

10 Obras, tomo XIV. 